\title{
Luminescence lifetime imaging with transparent oxygen optodes
}

\author{
Gerhard Holst*, Björn Grunwald \\ Max-Planck-Institute for Marine Microbiology, Microsensor Research Group, Celsiusstr. 1, D-28359 Bremen, Germany
}

\begin{abstract}
The imaging of two-dimensional (2D) solute distributions with planar optodes has become an important tool in biological and medical research. The development of versatile and flexible imaging systems, that enable both luminescence intensity and lifetime imaging, has generated various applications of planar oxygen optodes. Most of the applied optodes however, were not transparent. They either contained scattering particles in the sensing layer for signal enhancement and/or an optical insulation to separate the signal from ambient light. Since the modular luminescence lifetime imaging system (MOLLI) enables luminescence lifetime imaging, we used transparent planar oxygen optodes to investigate simultaneously the $2 \mathrm{D}$ distribution of oxygen and the structure that causes this distribution. This is done by either using the luminescence intensity images or different spectral illumination for structural imaging and the luminescence lifetime images for oxygen distribution imaging.

As the distribution of oxygen plays a key role at different spatial scales, we present results from applications of the transparent optodes to various biological systems: (a) to a coral sand sediment sample (macrolens application: resolution of approximately $50 \mu \mathrm{m}$ per pixel); (b) to a lichen with cyanobacteria as symbionts (endoscope application: resolution of approximately $15-62.5 \mu \mathrm{m}$ per pixel) and (c) to a foraminifer with diatoms as symbionts (microscope application: resolution of approximately $3.8 \mu \mathrm{m}$ per pixel). The results demonstrate the performance and some of the limits of the application of transparent optodes. Other possible fields of applications that are not restricted to marine environment are discussed. (C) 2001 Elsevier Science B.V. All rights reserved.
\end{abstract}

Keywords: Luminescence lifetime imaging; Fluorescence lifetime imaging; Oxygen optode; Planar optode

\section{Introduction}

Various publications in the last decade demonstrated that imaging of two-dimensional (2D) solute distributions with luminescent indicators has become an attractive tool in medicine, biology and physics. The first described image processing systems and experimental set-ups were designed and optimised for specific applications like measurements of oxygen distribution in tissue [1,11-13], $\mathrm{pH}$ and $\mathrm{Ca}^{2+}$ distributions in cells [3,14-16], oxygen partial pressure on skin surface or oxygen flux into skin $[5,17,18]$, oxygen distribution across the water-sediment interface $[9,10]$ and in biofilms [19]. However, many of these systems lack versatility because they were for example, confined to microscope set-ups [3,4,15,16,20-22]. Therefore, we suggested in 1995 a new modular luminescence lifetime imaging system (MOLLI [7]) that allows for versatile applications at a wide range of spatial resolutions. While many of the biological applications used planar oxygen optodes with optical

\footnotetext{
* Corresponding author. Tel.: +49-421-2028-834; fax: +49-421-2028-690.

E-mail address: gholst@mpi-bremen.de (G. Holst).
}

isolations $[9,10,19]$, we combined luminescence lifetime imaging with transparent sensing layers to open a second layer of information. By using the backscattered and reflected light if the sensing foil is illuminated by "white" light (broad spectral composition like halogen lamps) structural information can be recorded that in turn can be related to the oxygen distribution.

\section{Measuring system}

\subsection{Oxygen sensing}

The dynamic quenching of luminescence by oxygen is the principle for the measurement of oxygen distributions in multiple applications $[1,5,9,10,12,13,17,18,23-25]$ or of barometric pressure [2]. The sensors applied have a planar structure with the luminescent indicator embedded in PVC [26] that is spread on a transparent polyester support foil (Mylar, DuPont, USA) by a knife-coating process. After drying, the sensing film thickness was approximately $10 \mu \mathrm{m}$. The indicator [27-31] ruthenium(II)-tris-4,7-diphenyl-1,10phenanthroline, with trimethylsilylpropanesulfonate as the counter ion is widely used for oxygen sensing purposes. It 
has a high quantum yield and long luminescence decay times in the range of $\mu \mathrm{s}$, an absorption spectrum that almost perfectly overlaps with the emission spectrum of blue LED's $\left(\lambda_{\text {peak }}=475 \mathrm{~nm}\right.$, HLMP-CB15, Agilent, USA $)$ and a relatively large Stokes shift with an emission maximum at $605 \mathrm{~nm}$, that favours its application for luminescence lifetime imaging.

The sensor area is imaged through an optical emission filter (KV550, Schott, Wiesbaden, Germany) by lenses or imaging fibres onto the CCD-chip of a camera: each pixel on the chip is monitoring a small amount of the total emitted luminescence, that either correlates to the absolute intensity or, with an appropriate timing, to a part of the emitted light from the decay curve.

The oxygen optodes are calibrated with a two component model of the Stern-Volmer-equation [32,33] that has been modified in a way, that the second component is assumed to be non-quenchable. Since our characterisation measurements have shown that for a specific indicator-matrix mixture the ratio or fraction between these two components stays constant (frac has to be determined once). Therefore, the resulting procedure is a two-point calibration.

$\frac{\tau}{\tau_{0}}=\frac{I}{I_{0}}=\frac{\text { frac }}{\left(1-K_{\mathrm{SV}}\left[\mathrm{O}_{2}\right]\right)}+(1-$ frac $)$

where $\tau_{0}, \tau$ denotes lifetime in the absence or presence of oxygen, $I_{0}, I$ the luminescence intensity in the absence or presence of oxygen, $K_{\mathrm{SV}}$ the bimolecular quenching coefficient, $\left[\mathrm{O}_{2}\right]$ the oxygen concentration in vol.\% or percentage of air saturation, frac, the fractionating factor.

For image processing the variables $\tau_{0}, \tau, K_{\mathrm{SV}},\left[\mathrm{O}_{2}\right]$ are replaced by the corresponding images.

\subsection{Imaging system}

Luminescence lifetime imaging has two major advantages compared to conventional luminescence intensity imaging. It allows for a good contrast enhancement and for a background suppression if unwanted luminescence contributions are present in the image, e.g. chlorophyll fluorescence in biological samples. If the background luminescence has a different decay time than the luminescent indicator of interest, it is possible to separate both signals by lifetime imaging. Further, lifetime imaging does not depend on intensity variations due to inhomogeneous illumination, photobleaching (if the variations do not occur faster than the image recording) or variable indicator concentrations and calibration free sensing applications [13] can be achieved. The recently published modular luminescence lifetime imaging system consists (Fig. 1) of a thermoelectrically cooled CCD-camera that can be gated at minimum $\tau=500$ ns (SensiMod, PCO, Germany), a controlling PC and an external trigger unit (SG535, SRS, USA). Lifetime imaging [6-8,13,22,23], lifetime corresponding phase delay ratioing $[5,8,17,18]$ and intensity imaging are options of that system. We apply the pulse-gate (or rapid lifetime determination) method to record images that represent integral parts of the light of the luminescence decay curve.

The excitation light source, appropriate optical filters for excitation and emission, the planar optode and the optics that image the sensor to the CCD-chip are considered to be the application oriented part of the imaging system (Fig. 1, left frame). According to the spatial resolution that should be achieved this part of the imaging system has to be optimised.

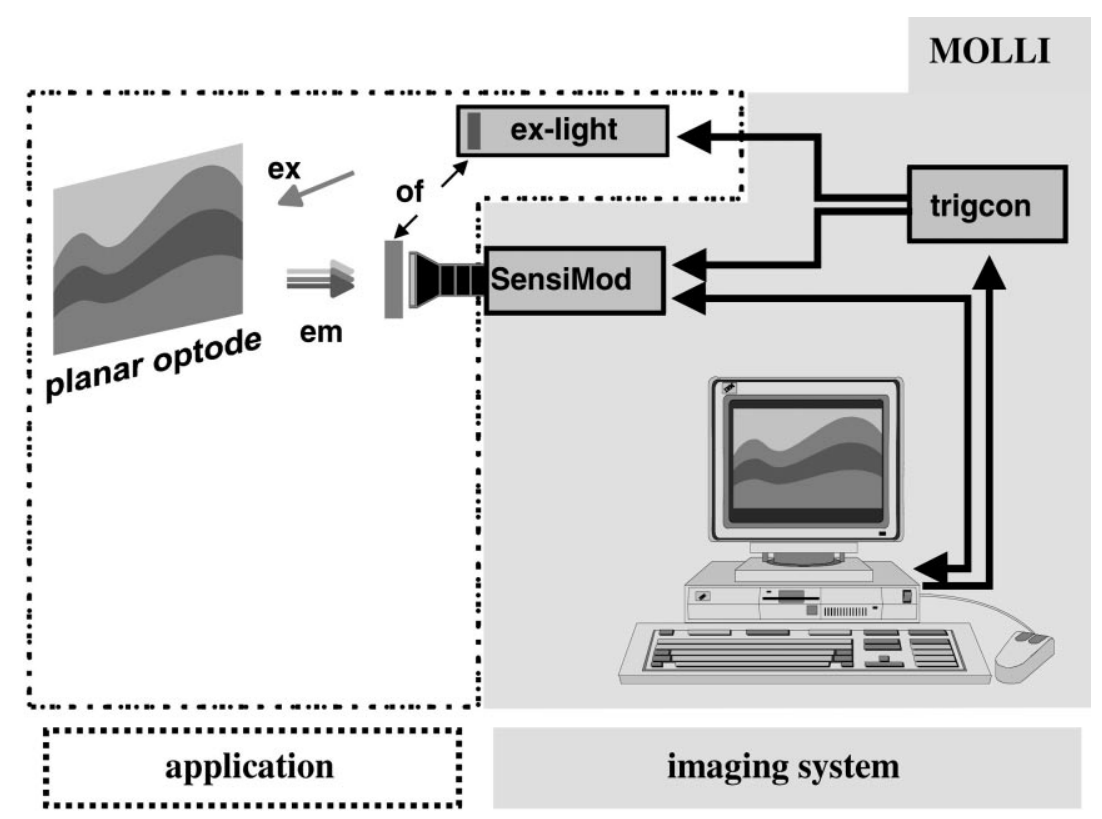

Fig. 1. Schematic overview of MOLLI. The imaging system consists of a fast gateable CCD-camera (SensiMod), a PC and a trigger control (trigcon). The application related part consists of the investigated sample and the planar optode, an excitation light source (ex-light), means to transport the light to the optode (direct or via light guide) and the emitted luminescence to the CCD-chip (macrolens, endoscope, microscope), and optical filters (of) if necessary. 
Table 1

\begin{tabular}{|c|c|c|}
\hline Application & Technical data & $\begin{array}{l}\text { Spatial resolution } \\
(\mu \mathrm{m} \text { per pixel) }\end{array}$ \\
\hline Macrolens Tevidon (Docter Optics, Germany) & Focal length $=25 \mathrm{~mm}$, aperture $=1.4$ & 48 \\
\hline Endoscope (Schölly Fiberoptic, Germany) & $\begin{array}{l}30000 \text { imaging fibres view angle }=45^{\circ} \text {, imaging fibre } \\
\text { bundle diameter }=0.91 \mathrm{~mm} \text {, outer diameter }=2 \mathrm{~mm}\end{array}$ & 24 \\
\hline $\begin{array}{l}\text { Microscope lens Plan-Neofluar (Axiovert } 25 \text { microscope, } \\
\text { Zeiss, Germany) }\end{array}$ & Magnification $=5$, aperture $=0.15$ & 3.8 \\
\hline
\end{tabular}

The presented experiments apply three different optical setups with corresponding spatial resolutions (Table 1).

Fig. 2 shows the relation of timing signals and the corresponding light signals to clarify the image recording structure. The camera has two ON/OFF signals. First, the integration time signal (integration time for 1 image window, Fig. 2), which is software controlled, determines whether the camera is ready to collect light, and can be adjusted in the range of $500 \mathrm{~ns}$ to $450 \mathrm{~ms}$. Second, the modulation input signal (TTL signal, hardware controlled) controls the electronical "shutter", that switches ON and
OFF the acceptation of light on the CCD-chip, but only if the first signal, the integration time, is ON.

But the electronical "shutter" is not a real shutter and has a limited blocking efficiency. That means it does not interrupt the light path, but directs the light induced charge carriers to the CCD-pixels or to an on-chip waste channel. Therefore, even for lifetime imaging an appropriate emission filter is helpful, because it attenuates the impinging light. Otherwise, if the impinging light passes over a certain level, light contributions in the recorded image may appear although the shutter is closed.

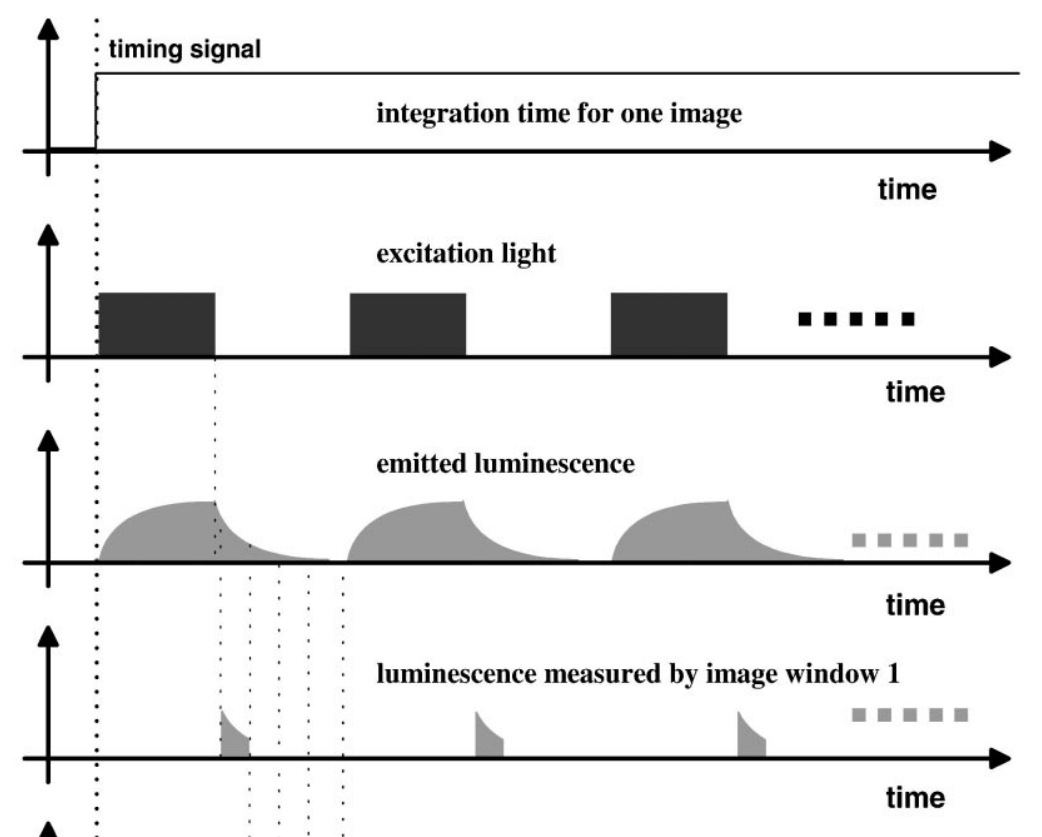

luminescence measured by image window 2

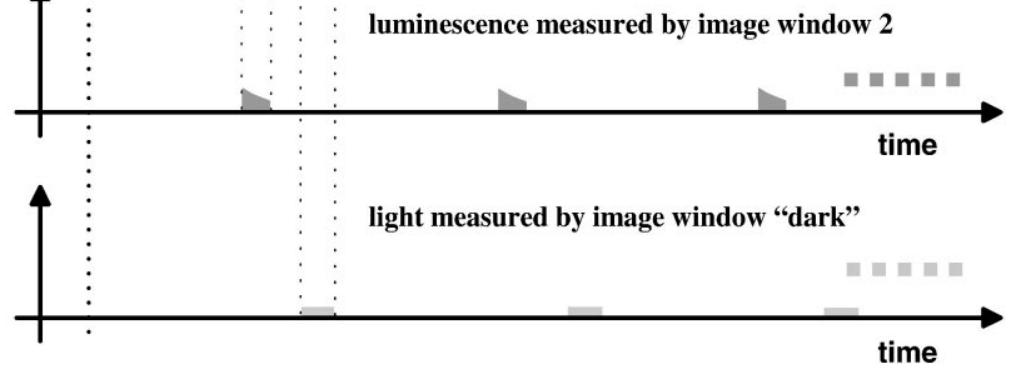

Fig. 2. Structure of timing and light signals. The general time window for an image is given by the integration time of the camera that turns the camera into a ready-to-accept-light state. Within this time frame a structure of events is repeated at a defined repetition frequency, for example, for image window 1: the excitation light is switched ON and OFF, consequently the luminescence follows with a certain delay in rise and decay, for image window 1 the camera is "opened" by the modulation signal for a time $\Delta_{i}$. This sequence is repeated (an example with real time values is given in the text). For image window 2 and the window "dark" the delays after switching OFF the excitation light are different but the width $\Delta_{i}$ is the same. 
The time values in the following explanation of the timing structure of the image recording represent actual values from the experiments. The integration time window for a set of images is adjusted for example, to $t_{\text {int }}=350 \mathrm{~ms}$. Within this time a couple of events are continuously repeated. Image window 1: the excitation light is switched $\mathrm{ON}$ (excitation light, Fig. 2) for $t_{\mathrm{ex}}=4 \mu \mathrm{s}$. With its delay, due to filling up and depleting the excited states, the luminescence light signal seen by a pixel follows (emitted luminescence, Fig. 2). For lifetime imaging, the modulation signal of the camera is switched ON at $\Delta_{1}=0.5 \mu$ s after switching OFF the excitation light, and switched OFF again after $\Delta_{i}=3 \mu \mathrm{s}$. At $t_{\mathrm{tot}}=11 \mu \mathrm{s}$ after first switching ON the excitation light, it is switched $\mathrm{ON}$ again. This is repeated as often as possible within the major integration time window. The repetition frequency can be calculated as

$f_{\text {mod }}=\frac{1}{t_{\mathrm{ex}}+\Delta_{1}+2 \Delta_{i}+\Delta_{2}+0.5 t_{\mathrm{ex}}}$

With the given time values above and a $\Delta_{2}=-1.5 \mu$ s for image window 2 this gives a repetition frequency $f_{\text {rep }}=90 \mathrm{kHz}$. That means that the described events are repeated 31,818 times and the according light signals are integrated on each pixel of the CCD-chip. When the integration time $t_{\text {int }}$ is over, the first image is read out of the camera. Then image window 2 (Fig. 2) is recorded the same way except that time width $\Delta_{1}$ is replaced by $\Delta_{1}+\Delta_{i}+\Delta_{2}$. Finally with the same modulation frequency but without excitation light ON (expressed in Fig. 2 by the shift of the image window "dark" into the time area where no light occurs) a "dark" image is recorded that contains ambient and background light information. If these light conditions do not change between the recording of the three image windows, it is possible to do lifetime imaging with some amount of ambient light present (which is not possible for intensity imaging). Before the images are stored, the image window "dark" is subtracted from image window 1 and image window 2 . Additionally a parameter text file is store that contains all timing and measuring information. The controlling software Look@Molli_v1.1 is developed and written in Delphi 4 (Inprise, USA).

\subsection{Image processing}

The program MolliView.pro for the processing of the recorded images is written in IDL (Research Systems Inc., USA) and enables the complete processing and conversion of all images of all available measuring schemes (intensity, lifetime 2 and 3 windows, phase delay ratio) that are implemented in the image recording program. The general path of image processing with 2 window lifetime imaging is shown in Fig. 3. Every measurement consists of a set of three files as mentioned above. To convert measured image sets, two calibration image sets are necessary, for example one at zero oxygen and one at air saturation. Each set is converted into $2 \mathrm{D}$ arrays of real values of lifetimes by the following calculation. It is assumed that the decay curve of the luminescence can be described by a monoexponential decay with an apparent lifetime $\tau$ as characteristic time parameter

$I_{i}=I_{0} \tau \exp \left(\frac{t_{i}}{\tau}\right)\left[1-\exp \left(-\frac{\Delta_{i}}{\tau}\right)\right]$

where $I_{0}$ is the intensity when the excitation light is switched OFF, $I_{i}$ the intensity at time $t_{i}$.

The decay curve integration time $\Delta_{i}$ is constant for image window 1 and 2 . Therefore, the lifetime conversion calculation is reduced to

$$
\tau=\frac{\Delta_{i}+\Delta_{2}}{\ln (\text { image window } 2 / \text { image window } 1)}
$$

where $\Delta_{i}$ is the decay curve integration time, $\Delta_{2}$ the time difference between end of decay curve integration time for image window 1 and start of image window 2 .

It takes less calculation time than the 3 window approach [7] but gives sufficiently good results [6]. When each image set is converted into lifetime array images, the two calibration images are used to calculate a $K_{\mathrm{Sv}}$ array image like the calibration equation above. Now the zero lifetime image and the $K_{\mathrm{SV}}$ image are used to calculate, with respect to the above modified Stern-Volmer-equation, oxygen images in units that have been used for calibration. In the present applications it is percentage of air saturation. The resulting oxygen array image can be colour coded and scaled for better visualisation purposes (Fig. 3).

Each position of the image represents a real value of the oxygen concentration. Therefore, model calculations with profiles across the image as well as other investigations that request high spatial resolution information of the oxygen distribution can be performed. Compared to single profile measurements with microoptodes [29,34] a $640 \times 480$ pixel image contains maximum 640 oxygen profiles instead of one.

\section{Experimental}

All applications presented in the following investigate the oxygen production and respiration of photosynthetic active organisms or communities at different spatial scales. These organisms like cyanobacteria or diatoms produce and consume oxygen while they are illuminated with light. In the darkness there is only consumption. To investigate the conditions of their metabolism, a usual procedure is to illuminate a sample that is preserved under nature like conditions with a homogeneous light field. After approximately $30-40$ min they reach a steady state, then the light is switched OFF. The fast reactions in oxygen distribution as well as the long-term reaction until the dark steady state is reached, give characteristic values for production and consumption of the organisms.

The optodes that we applied were transparent. Therefore, the sunlight simulating illumination of the sample with its 


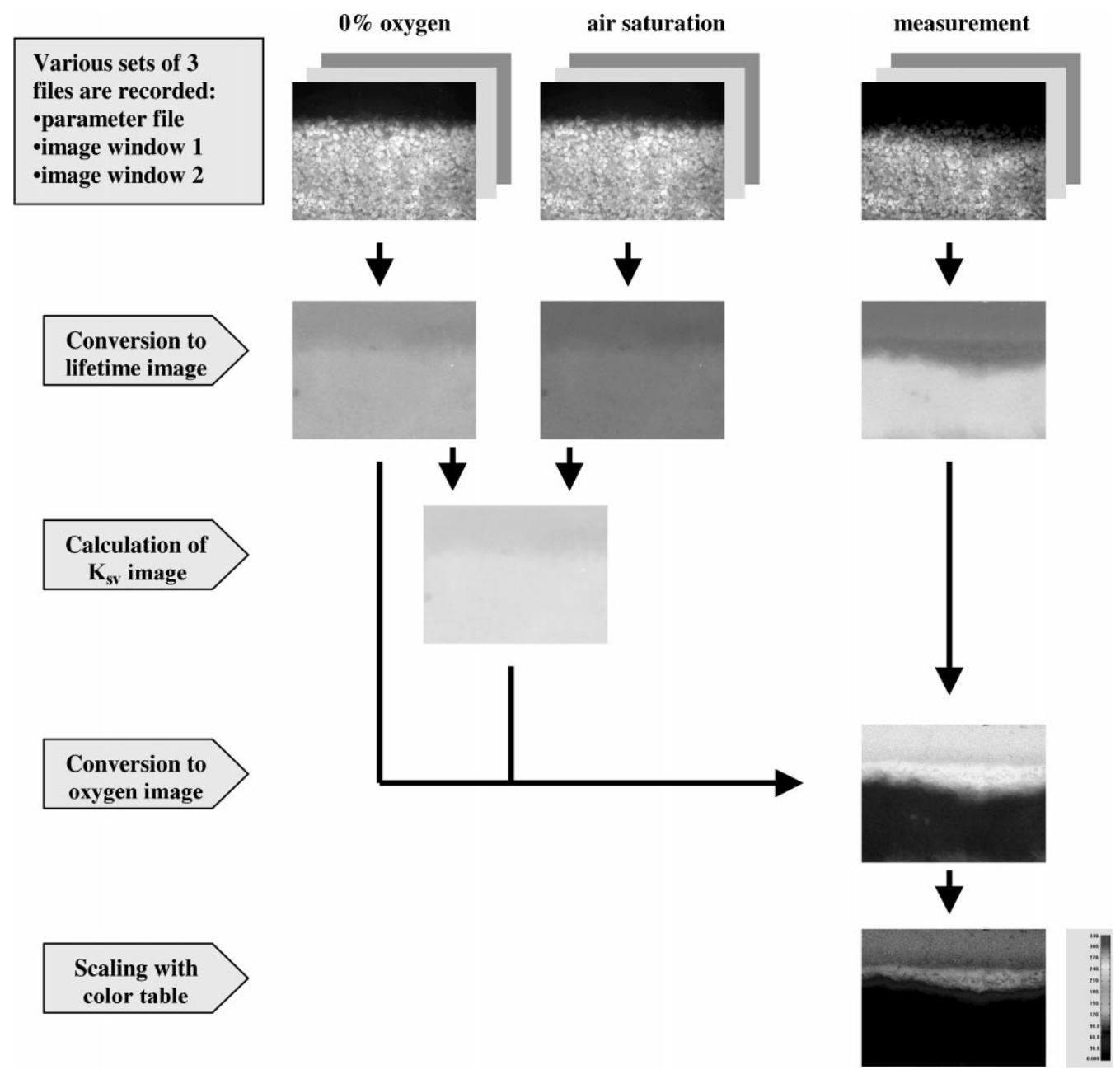

Fig. 3. Structure of image processing. Various sets of three files are recorded. Two calibration sets and numerous sets of measurements. After calculation of the corresponding luminescence lifetimes, each pixel representing a former measured amount of light is then converted into a real time value in a $640 \times 480$ array. These values are used for further conversion into oxygen values that can be visualised by an image like display (for more detailed explanation of image processing see text).

high light level would drive the described image window "dark" already in an overflow situation. The real measurement was only started when the sunlight simulating illumination was switched OFF.

\subsection{Macrolens application}

If photosynthetic active shallow water sediments are investigated spatial resolutions between 50 and $100 \mu \mathrm{m}$ are sufficient. This can be achieved by the use of a macrolens and optional distance rings. A coral sediment sample from Shark Bay, Heron Island (Great Barrier Reef, Australia) is placed in a flow chamber where in advance a transparent oxygen optode was fixed to the transparent side wall (Fig. 4[A], optode/support foil/glass wall). The sample when inserted into the flow chamber is moved gently to the foil to reach a tight contact between sediment and optode. The sediment is flushed at a constant flow with aerated water taken from the sample site. The set-up was situated in a dark room to enable control of the light conditions with a halogen lamp. The excitation light source consists of a self-constructed "light frame" of 18 LED's (same as above). In front of the LEDs holographic $60^{\circ}$ diffusers are mounted to generate a reasonably homogeneous light field. The emitted luminescence passes through the frame, a KV550 filter and is imaged via the above mentioned lens at a resolution of approximately $46 \mu \mathrm{m}$ per pixel.

The sample was illuminated at a constant light energy level for $35 \mathrm{~min}$ with a halogen lamp. Then the illumination was switched OFF. For 2 min images were recorded at a high rate (1 image set per $4 \mathrm{~s}$, because of average 4 ) and then for 6 min images were recorded at a low rate (1 image set per minute). This was repeated for increasing light energy levels. Finally after a longer period of darkness $(6 \mathrm{~h})$ the chamber was illuminated from the side by a halogen lamp (Fig. 4[A], sample illumination) to record structure intensity images. For calibration at the end of the experiments the sample was removed. The chamber was filled with stagnant 
[A]

optode I support foil I glass wall

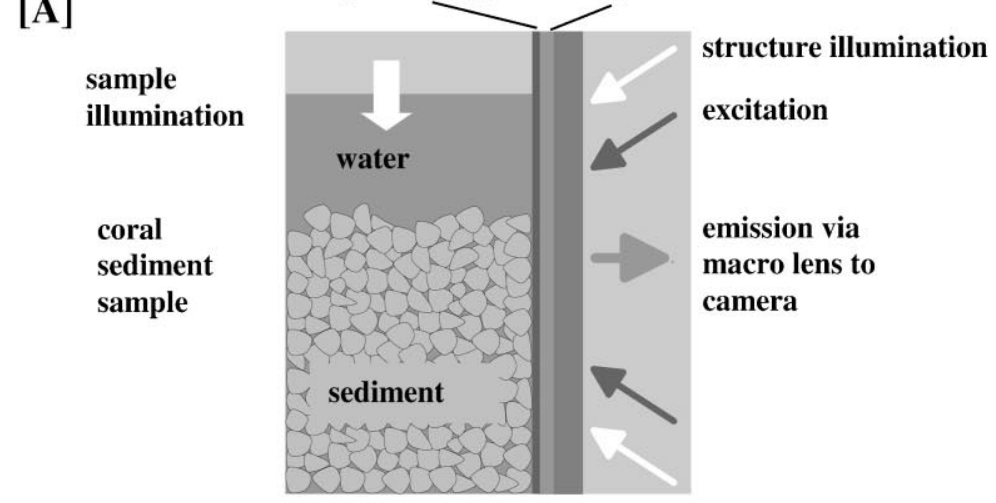

$[B]$

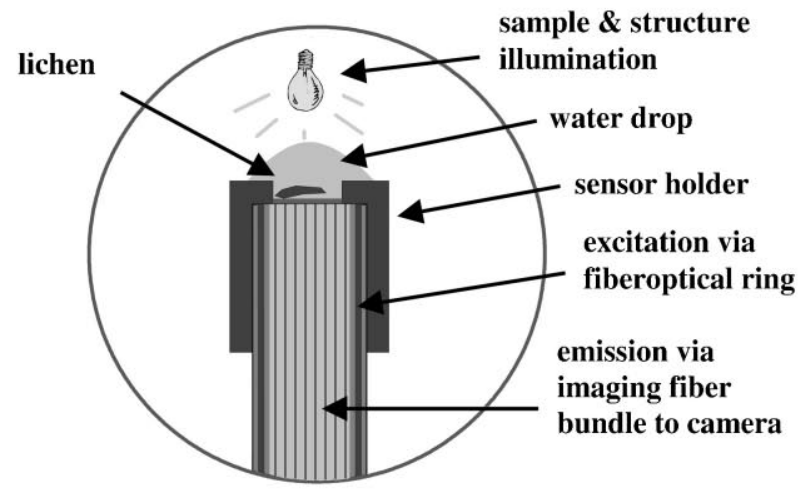

[C]
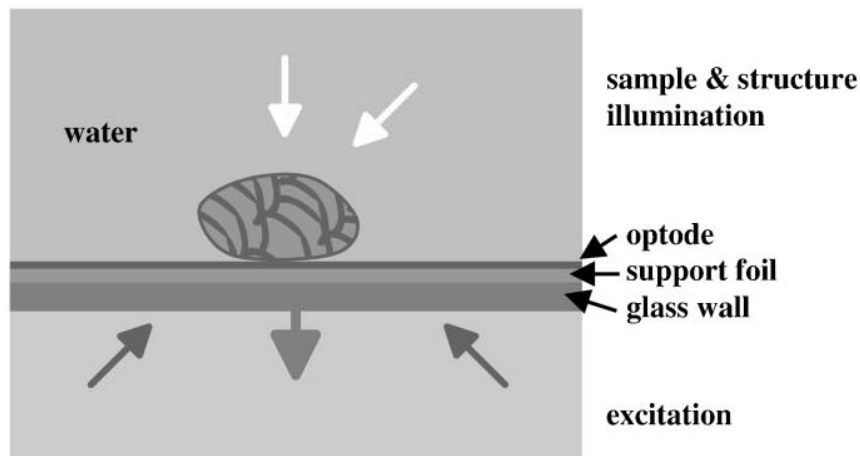

emission via microscope

lens to camera

Fig. 4. Schematic drawings of the applications. (A) The transparent optode (optode and support foil) is fixed to a flow chamber window (glass wall, thickness $=2 \mathrm{~mm}$ ). The coral sand sediment sample touches the optode. The luminescence image view is through the macrolens of the CCD-camera. The excitation light (blue LEDs, $\lambda_{\text {peak }}=475 \mathrm{~nm}$ ) and the illumination for structure images (halogen lamp) are positioned like the camera on the right side. A collimated halogen lamp from above illuminates the sample (sample illumination). (B) A piece of soil lichen is placed on top of a transparent planar optode, which is separated from the endoscope by a $2 \mathrm{~mm}$ thick polycarbonate window. This lichen is then covered with a water drop (diameter approximately $5 \mathrm{~mm}$ ) and illuminated by a halogen lamp (sample and structure illumination). The luminescence image view is through the imaging fibre bundle in the core of the endoscope, while the excitation is guided through the fibreoptical ring of the endoscope (six blue LEDs, $\lambda_{\text {peak }}=475 \mathrm{~nm}$ ). (C) The foraminifer (Amphistegina lobifera with diatoms as symbionts) is placed on top of a transparent optode (optode and support foil), which itself is fixed at the bottom of a petri-dish (glass wall). The luminescence image view is through the lens of the inverse microscope from below. The optode is excited from below (blue LEDs, $\lambda_{\text {peak }}=475 \mathrm{~nm}$ ), while the sample (halogen lamp) and structure (microscope lamp) illumination is from above.

water, which was flushed with either nitrogen or air until no change in the lifetime values of the recorded images could be observed anymore (approximately $30 \mathrm{~min}$ ). All images were recorded without binning and with an average of 4 images.

\subsection{Endoscope application}

This was a functional test. On top of the endoscope (Fig. 4[B]) a polycarbonate plate with an attached transparent 
oxygen optode on its upper surface was mounted (fixation with silicone). On this optode a dry piece of a soil lichen Collema sp. (Utah, USA), a symbiosis of a fungus and the cyanobacterium Nostoc, was placed. Then a large water drop was deposited to cover the lichen and the optode. A halogen lamp (Fig. 4[B], sample and structure illumination) illuminated the whole set-up for approximately $30 \mathrm{~min}$ at a constant light level to reach a steady state of oxygen production and consumption. Then the illumination light was switched OFF. For 20 min image sets were recorded at 1 image set per minute. Then the sample was illuminated again to record a structure image (Fig. 4[B], sample and structure illumination). Finally water and lichen were removed and two calibration image sets were recorded, one with an air saturated and one with a deaerated water drop. All images except the structure image (image recorded without binning resulting in a resolution of $16.2 \mu \mathrm{m}$ per pixel) were measured with a pixel binning of 2 , which according to the distance of the optode from the endoscope surface reduced the possible resolution to approximately $62.5 \mu \mathrm{m}$ per pixel.

\subsection{Microscope application}

The transparent optode was fixed at the bottom of a petridish, which was filled with salt water at usual salinity for the benthic growing foraminifer Amphistegina lobifera that has diatoms as symbionts. Then a foraminifer was placed in the middle of the petri-dish and the dish was placed on the stage of an inverse microscope (Fig. 4[C]). The optode was excited by four LED's (type like above) that were circularly arranged (emitting upward) around the microscope lens. The emission was imaged by the microscope lens and adapting optics onto the CCD-chip. A halogen lamp from above (Fig. $4[\mathrm{C}]$, sample and structure illumination) illuminated the petri-dish from above for photosynthesis. The structure images were recorded between series of measurements by using the microscope lamp.

The experiments were similar to the procedure with the coral sediment sample except that the water was stagnant and not actively aerated. The foraminifer was illuminated for $35 \mathrm{~min}$ at a constant light energy level, then for $2 \mathrm{~min}$ images were recorded at 1 image set per second and for
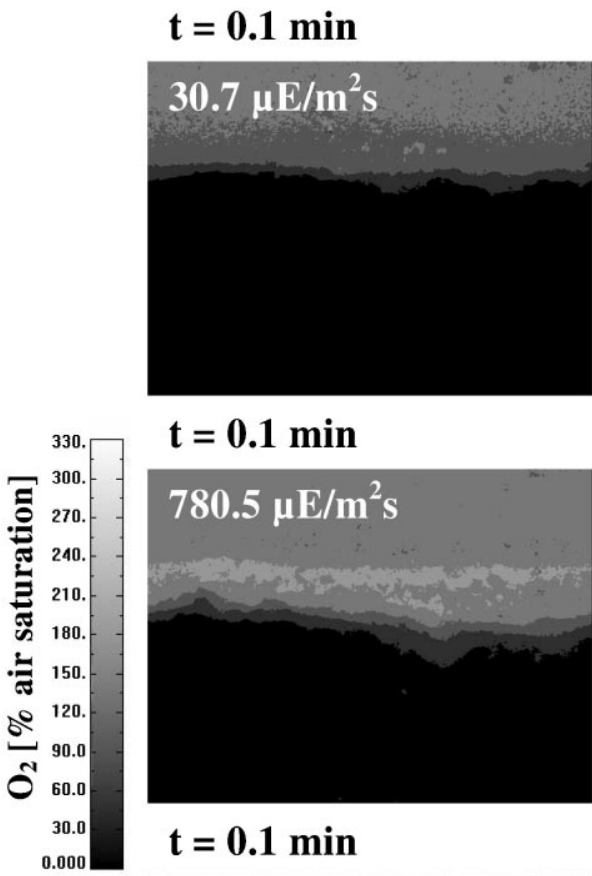

$$
\mathrm{t}=\mathbf{0 . 1} \mathrm{min}
$$
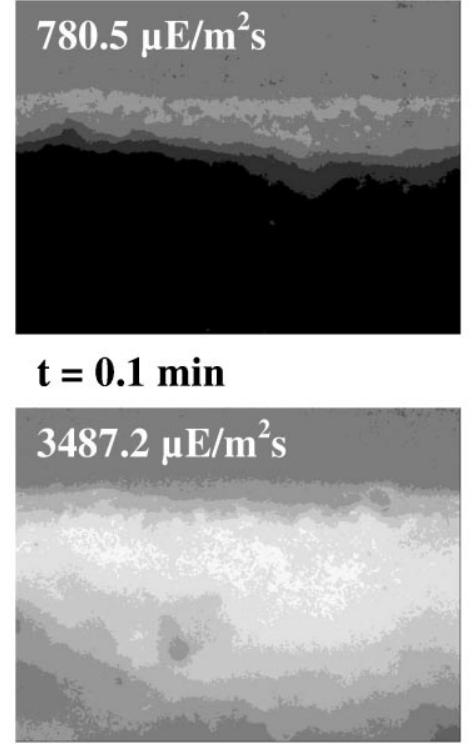

\section{$\mathrm{t}=\mathbf{5 . 2 2} \mathrm{min}$}

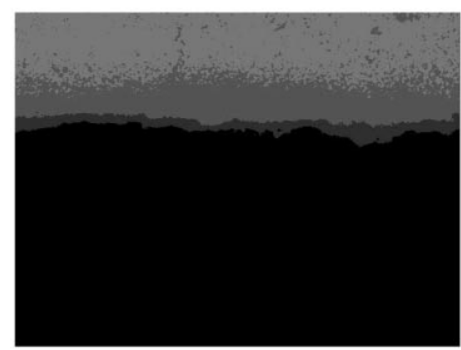

$$
\mathrm{t}=\mathbf{5 . 2 3} \mathrm{min}
$$

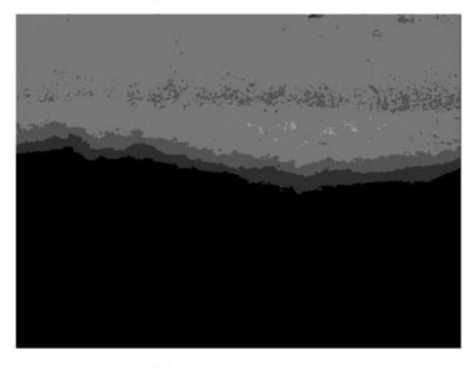

$t=8.23 \mathrm{~min}$

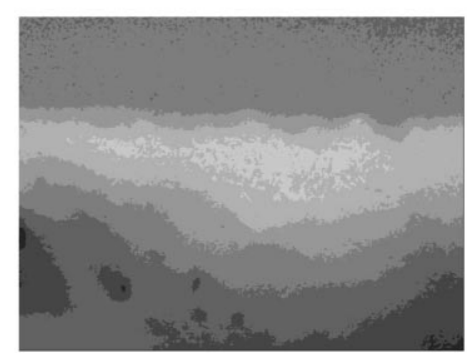

Fig. 5. Results of macrolens application. For each row of oxygen images the sediment sample was illuminated before at a constant light energy level (30.7, 780.5 and $3487.2 \mu \mathrm{E} / \mathrm{m}^{2} \mathrm{~s}$ ) for about $35 \mathrm{~min}$. Then the illumination was stopped and image series were recorded of which two images are shown. The times $t$ given above each image are related to the illumination stop. The oxygen levels have been divided into 11 ranges for better visualisation in grey coding. The grey level bar shows continuous scaling with ticks that correspond to the grey level in the distribution image. 
25 min at 1 image set per minute. This was repeated for increasing light energy levels. At the end of the experiments the foraminifer was removed and calibration images with air saturated and deaerated water were recorded.

\section{Results and discussion}

\subsection{Macrolens application}

Fig. 5 shows some result images of the coral sediment experiments. For light energy levels of: 30.7, 780.5 and $3487.2 \mu \mathrm{E} / \mathrm{m}^{2} \mathrm{~s}$ (measured with a calibrated light probe from Biospherical, USA) two samples of oxygen distribution images in unit percentage of air saturation after switching OFF the illumination light are given (Fig. 5, grey scale bar). The first column of images was recorded $0.1 \mathrm{~min}$ after switching OFF the illumination and the second column was recorded between 5 and 8 min after switching OFF. The images have been smoothed by a running average of 2 . The averaging reduces the spatial resolution to $96 \mu \mathrm{m}$ per pixel, which we considered sufficient, compared to standard profiling measurements with microoptodes at $100 \mu \mathrm{m}$ stepwidth. The grey scale coded images are divided into 11 ranges for better visualisation while the grey scale bar shows continuous values (in colour coding that we usually use for evaluation, continuous scaling is used).
After illumination at the lowest level the oxygen distributions short or long time after switching OFF the light do not show any significant difference or change. Therefore, it can be assumed, that the photosynthetic active organisms did not get enough light energy to produce mole oxygen by photosynthesis than they respire. The gradient from air saturated water that flows above the sediment surface, down to zero oxygen $1 \mathrm{~mm}$ below the surface is visible. The rest of the sediment, although the sediment grains were large and advective transport should be easier (grain diameters between 0.8 and $1.4 \mathrm{~mm}$ ), is anoxic. After illumination at medium light level, the oxygen production in the upper part of the sediment appears with oxygen concentration values between 150 and $180 \%$ air saturation. This zone decreases significantly within $5 \mathrm{~min}$. Furthermore a larger penetration depth for oxygen can be seen. This effect is even more pronounced after illumination at the highest light energy level. Here oxygen reaches peak values of more than $300 \%$ air saturation and penetration depths down to $2.4 \mathrm{~cm}$. The overall values are decreased within $8 \mathrm{~min}$ but still oxygen reach depths where it usually does not occur.

These final results could not be confirmed by microsensor measurements in the middle of the sample. The explanation for this discrepancy is the transparency of the optode (Fig. 6), that allows for light transmission in both directions.

While the sample is illuminated with the sun simulating light of the halogen lamp, part of this light passes through the
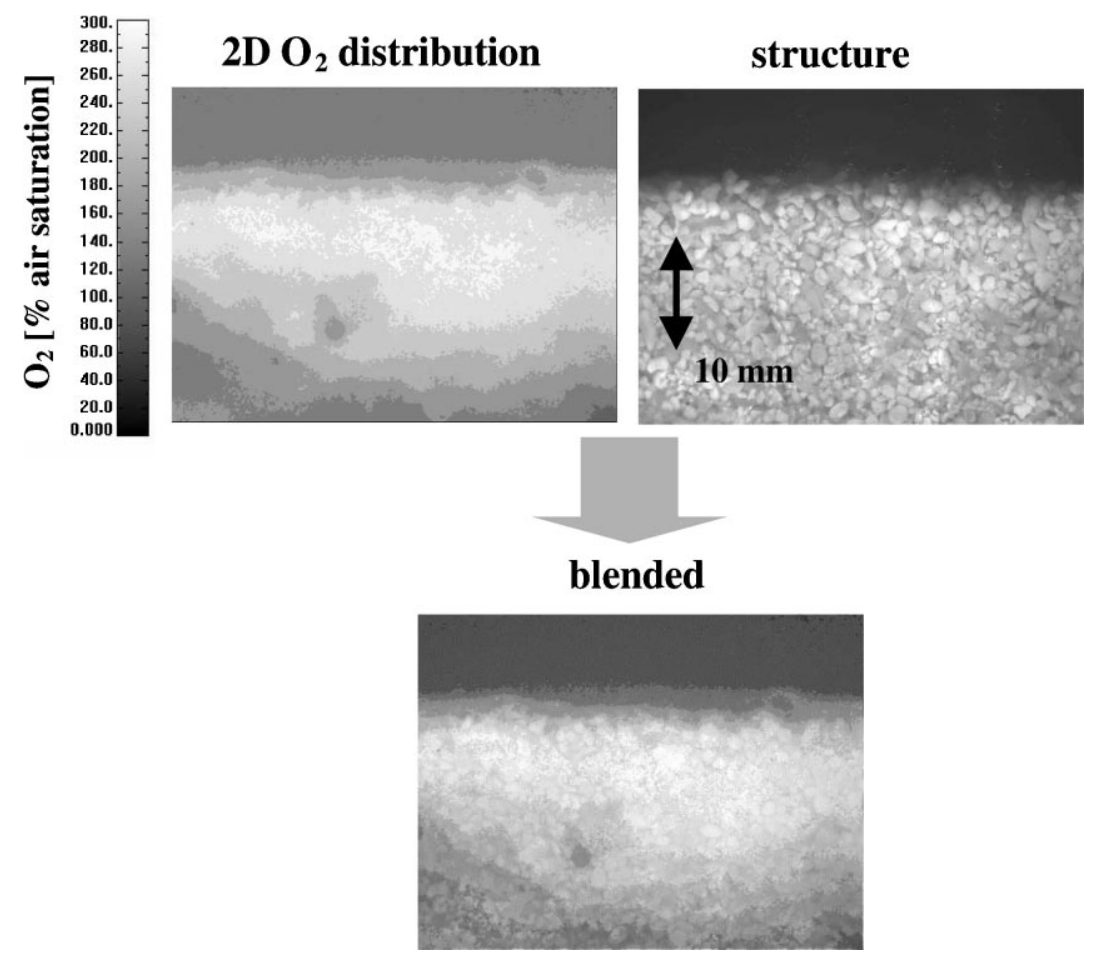

Fig. 6. Combination of structure and 2D oxygen distribution. The upper left image represents a visualisation of an oxygen distribution (lower left image of Fig. 5, for better visualisation the oxygen image has been divided into 11 ranges) and the upper right image shows the structure of the coral sand sediment sample (seen though the planar optode). Both can be blended together in a way that one image (here the oxygen distribution) becomes semi-transparent. Now each oxygen value can be linked to the location in the sediment structure. 


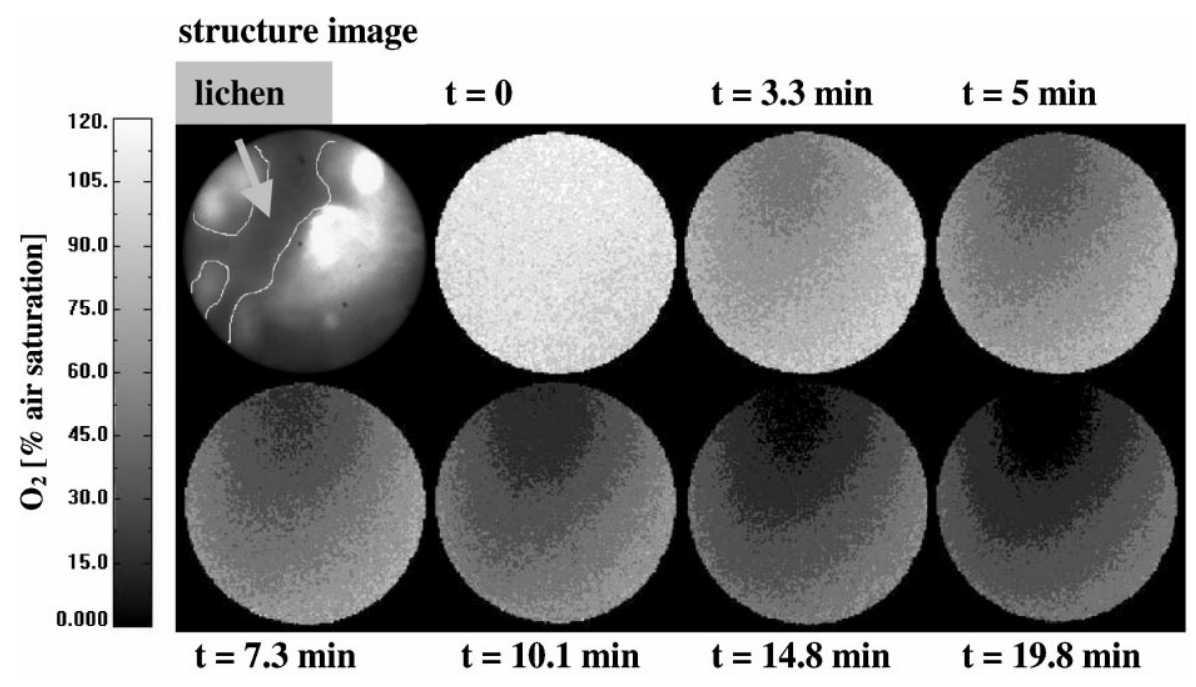

Fig. 7. Results of endoscope application. A structure image of the lichen in front of the endoscope and time series of oxygen distribution images is shown. The lichen Collema sp. with its symbionts was illuminated for $20 \mathrm{~min}$ and then the illumination was stopped. The image recording started $1 \mathrm{~min}$ after. The time $t$ given above each image therefore, relates to illumination stop plus $1 \mathrm{~min}$. The oxygen levels have been divided into eight ranges for better visualisation in grey coding. The grey level bar shows continuous scaling with ticks that correspond to the grey level in the distribution image.

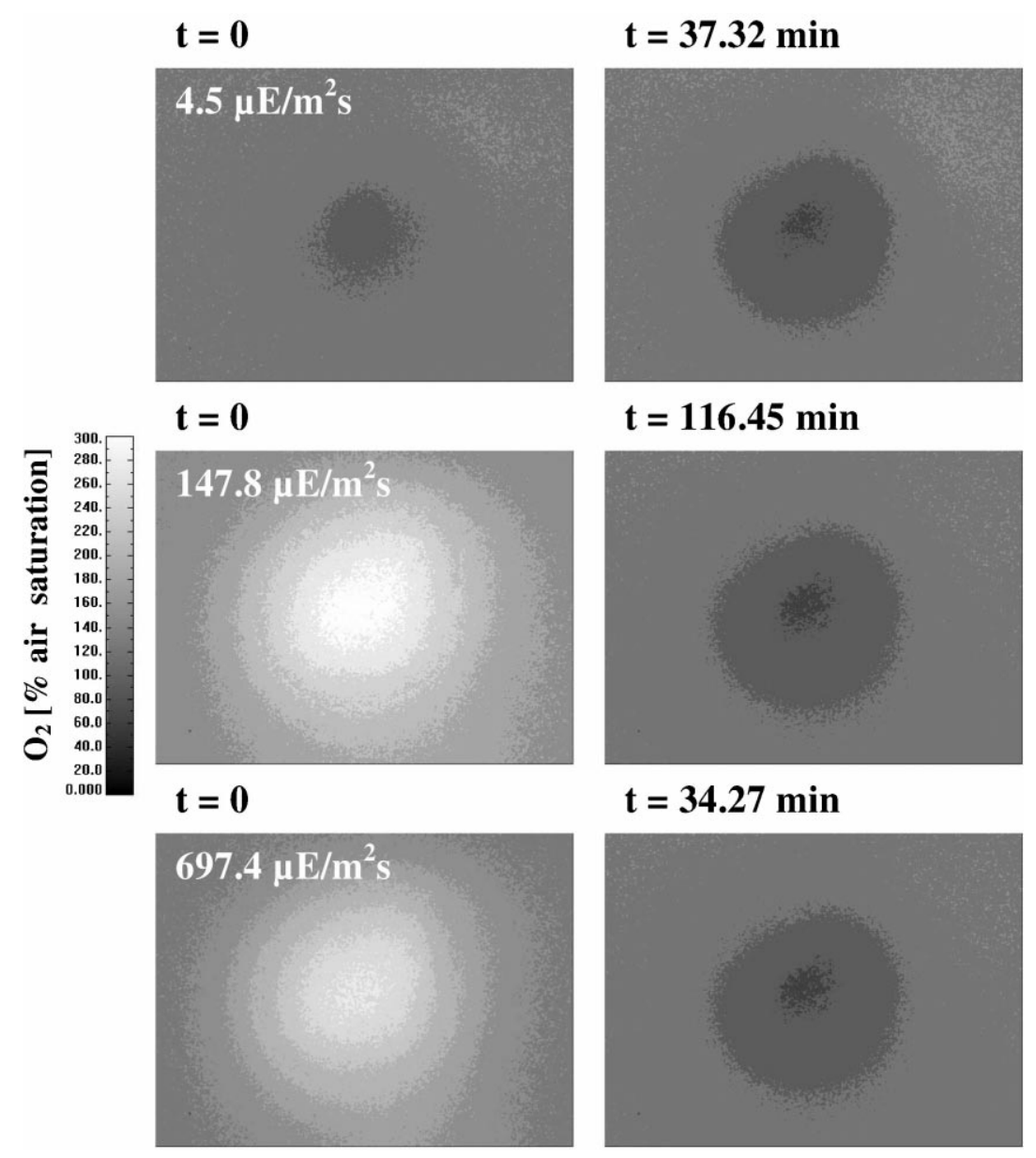

Fig. 8. Results of microscope application. For each row of oxygen images the foraminifer was illuminated before at constant light energy levels $(4.5,147.8$ and $697.4 \mu \mathrm{E} / \mathrm{m}^{2} \mathrm{~s}$ ) for about $35 \mathrm{~min}$. Then the illumination was stopped and image series were recorded of which two images are shown. The times given above each oxygen distribution image relate to the illumination stop. The oxygen levels have been divided into 15 ranges for better visualisation in grey coding. The grey level bar shows continuous scaling with ticks that correspond to the grey level in the distribution image. 
optode, is reflected at the sample chamber window and illuminates deeper layers of the sediment, which under natural conditions are not reached by this amount of light. In deeper layers there might be inactive organisms that are able to do photosynthesis, but do not have oxygen to respire or light to produce oxygen. They wait to be transported upwards with the next change of high and low tide. If so they would get light and immediately would start producing oxygen. This effect, as can be seen in Fig. 5 lowest row, is most pronounced at highest light levels. As possible solution for next applications a foil curtain down to the sediment surface during illumination could cover the window inside the chamber.

Nevertheless Fig. 6 demonstrate the important feature of the transparent optode. Structural images can be taken and the oxygen distribution images can be blended onto them. Now with the appropriate grey or colour scale coding (in colour it is more visible) it is possible to relate the oxygen concentration directly to the place where it occurs.

\subsection{Endoscope application}

The endoscope, which has been used for the first tests, had the wrong working distance. Therefore, the structural image of the lichen in Fig. 7 is not as sharp as it could be. The spatial resolution of the oxygen distribution images has been reduced to $62.5 \mu \mathrm{m}$ per pixel by pixel binning of 2 (the values of 2 pixel in $x$ and $y$ direction are summed to represent a single, binned new pixel value) like described above. The display of the oxygen values was divided into eight ranges for better visualisation in grey scale.

If the desert lichen Collema sp. (Utah, USA) is covered with water and illuminated with sunlight or similar the cyanobacteria Nostoc that live in this symbiosis immediately start their photosynthetic activity. Since the sample was illuminated for more than $30 \mathrm{~min}$, the cyanobacteria oversaturated the water drop with oxygen because they produce more oxygen than they consume. When the illumination stops, they instantly stop producing oxygen but continue respiring, so oxygen is depleted in the water drop. As the dimensions and water volume are very small in the drop, the net consumption of the organisms and therefore, the depletion is very fast. Hence, it was not possible with a master integration window of $400 \mathrm{~ms}$ (approximately $1.6 \mathrm{~s}$ for 1 image set) to see an oxygen production peak, that would correspond to the depletion peak that appears along the series in the upper half of the images (Fig. 7).

From the time series of oxygen distribution images the consumption of oxygen can be followed. The development of an oxygen depletion peak in the upper half of the endoscope images shows that parts of the lichen have been nearer to the optode surface or even touched it.

\subsection{Microscope application}

Fig. 8 shows results for the oxygen distributions at the bottom of the foraminifer Amphistegina lobifera. For light energy levels of: $4.5,147.8$ and $697.4 \mu \mathrm{E} / \mathrm{m}^{2}$ s (measured

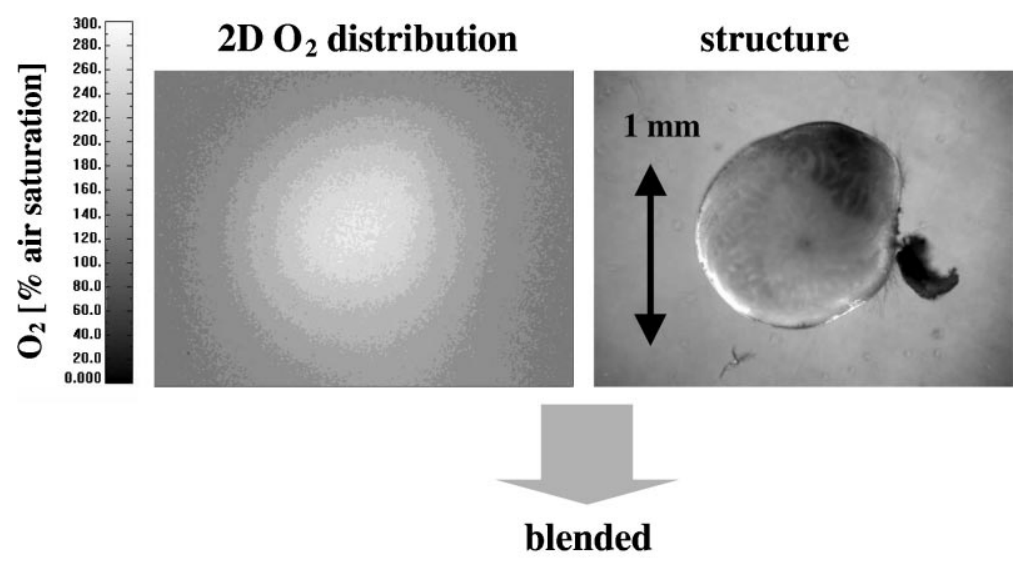

Fig. 9. Combination of structure and 2D oxygen distribution information. The upper left image represents a visualisation of an oxygen distribution (lower left image of Fig. 8, for better visualisation the oxygen image has been divided into 15 ranges) and the upper right image shows the structure of the foraminifer (seen though the planar optode). Both can be blended together in a way that one image (here the oxygen distribution) becomes semi-transparent. Now each oxygen value can be linked to the location at the bottom of the foraminifer. 
with a calibrated light probe from Biospherical, USA) two images of the oxygen distribution in unit percentage of air saturation are given (Fig. 8, grey scale bar). The images have been smoothed with a running average of 2 and thereby the spatial resolution has been reduced to approximately $7.6 \mu \mathrm{m}$ per pixel. The images in the first column were recorded directly after stopping the illumination. While the images of the second column were recorded after a period longer than $30 \mathrm{~min}$, which can be considered as dark steady state. The grey scale coded images are divided into 15 ranges for better visualisation while the grey scale bar shows continuous values (in colour coding that we usually use for evaluation, continuous scaling is used).

Depending on the light energy level they receive, the diatoms that live in symbiosis with the foraminifer Amphistegina lobifera start if illuminated producing more oxygen than they respire. This causes even at low light levels an increase of oxygen in the near environment compared to the dark steady state which shows a decreasing gradient in oxygen concentration from the outer parts of the foraminifer to the centre (Fig. 8, right column of distribution images). The opposite gradient can be seen from the medium to the highest illumination light level shortly after stopping the illumination. Here oversaturation levels of more than $260 \%$ air saturation are reached. While the right column of oxygen distribution images show the dark steady state situation.

The combination of the structural image of the foraminifer with the grey level scaled oxygen distribution image links the peaks in oxygen oversaturation and depletion to the centre of the optode touching foraminifer (Fig. 9). Now it is possible to measure oxygen concentrations even below the foraminifer that usually as a benthic growing organism is approached by microsensors from above. Furthermore various tests are now possible that investigate the balance of oxygen production and consumption versus change in environmental parameters.

\subsection{Limitations}

Backscattered or reflected luminescence from surfaces or particles behind the transparent optode might contribute to the recorded images. Therefore, care has to be taken that behind the optode there is no movement between the recording of each image window of a set. Otherwise the calculation of the luminescence lifetime in these images will partly fail and produce strange and false oxygen values.

Furthermore as can be seen in the macrolens application with the coral sediment sample, the scattering properties of the sample give different absolute light values within the image (compare the lower part with sand as background and the upper part with water as background). This results in highly different signal-to-noise-ratios within the same image. For example, the average pixel value (luminescence intensity) in the water phase was 500 compared to 2100 in the sediment. If the overall integration time is adjusted for a corresponding experiment, a compromise has to be taken between large possible values in the water phase and no overflow in the sediment area. The different levels of oxygen concentrations that determine the absolute luminescence intensity further complicate the situation. If samples are illuminated, light may pass through the optode and can illuminate regions of the sample at energy levels that are not reached in natural environments. The artificial light situation can result in strange oxygen distributions that have no relation to natural conditions. This only could be a problem for biological applications, where photosynthetic active organisms are involved, but it has to be considered in the planning of experiments. Furthermore, depending on the organisms involved in the experiments, excitation light reaches only attenuated by the optode the sample. If the spectrum of the excitation light overlaps with the absorption of the organisms, oxygen will be generated. The effect can be significant, as results (not shown) with high image recording rates in the microscope experiments with the foraminifer have shown. This can be overcome by change of excitation light source and an optional change of the oxygen indicator, e.g. phosphors [13,35,36].

\section{Conclusion}

The combination of luminescence lifetime imaging with transparent planar oxygen optodes offer for the first time in biological applications a direct link between the 2D oxygen distribution to the structural information of an image of the investigated sample. In earlier applications with optically isolated oxygen optodes this relation had to be determined by additional experiments like the appearance of zero oxygen dots in the 2D distribution caused by granules of an oxygen scavenger that were deposited on the sediment surface $[8-10,18,19]$. The presented results prove the excellent performance of the MOLLI system together with its high flexibility in applications at various spatial resolutions (from 3.8 to $62.5 \mu \mathrm{m}$ per pixel).

However, the results also revealed some limitations of the application of transparent optodes. They are due to the fact that the transparency of the planar optodes is not restricted to the light signal of interest. Excitation light reaches the sample and even light of the general illumination of the sample may reach parts of the sample that are not illuminated under natural conditions. The possible consequent artefacts occur mainly in applications where photosynthetic active biological systems are investigated and have to be considered. Solutions can be in optimised set-ups (additional shutter) or in optimised indicators that allow for excitation wavelengths that are not as efficiently absorbed by the organisms.

Nevertheless the application of transparent optodes with MOLLI is not confined to these very specific biological systems. There are numerous application fields in other biological systems, in geological and environmental systems as well as in medicine. 
Another feature of this measuring system that will be investigated is the option of additional spectral measurements through the planar optode, which could give further insight into the biological processes within the biological system. Furthermore previously published detection schemes [37] and new indicators for even other parameters than oxygen [38,39] can be applied with the imaging system MOLLI and used with transparent sensors to enable similar measuring strategies for other relevant parameters.

\section{Acknowledgements}

The authors wish to thank Michael Kühl, Ferran GarciaPichel and Stephanie Köhler-Rink for access to the biological samples and valuable discussions about the interpretation of the results. Furthermore Ingo Klimant and Gisela Hirlmeier are thanked for the preparation of the transparent optodes and valuable discussions about their characteristics. Finally the Max-Planck-Society is thanked for financial support.

\section{References}

[1] W.L. Rumsey, J.M. Vanderkooi, D.F. Wilson, Imaging of phosphorescence: a novel method for measuring oxygen distribution in perfused tissue, Science 241 (1988) 1649-1651.

[2] J. Kavandi, J. Callis, M. Gouterman, G. Khalil, D. Wright, E. Green, D. Burns, B. McLachlan, Luminescent barometry in wind tunnel, Rev. Sci. Instrum. 61 (1990) 3340-3347.

[3] J.R. Lakowicz, K.W. Berndt, Lifetime-selective fluorescence imaging using an RF phase-sensitive camera, Rev. Sci. Instrum. 62 (1991) $1727-1734$.

[4] G. Marriott, R.M. Clegg, D.J. Arndt-Jovin, T.M. Jovin, Time resolved imaging microscopy - phosphorescence and delayed fluorescence imaging, Biophys. J. 60 (1991) 1374-1387.

[5] P. Hartmann, W. Ziegler, G. Holst, D.W. Lübbers, Oxygen flux fluorescence lifetime imaging, Sens. Actuators B 38 (1997) $110-115$.

[6] G. Holst, B. Grunwald, I. Klimant, M. Kühl, A luminescence lifetime imaging system using imaging fibers to measure the $2 \mathrm{D}$ distribution of $\mathrm{O}_{2}$ in biological samples, in: Proceedings of the SPIE Conference on Fibre Optic Sensor Technology and Application, Vol. 3860, 1999, pp. 154-163.

[7] G. Holst, O. Kohls, I. Klimant, B. König, M. Kühl, T. Richter, A modular luminescence lifetime imaging system for mapping oxygen distribution in biological samples, Sens. Actuators B 51 (1998) 163170.

[8] G. Liebsch, I. Klimant, B. Frank, G. Holst, O.S. Wolfbeis, Luminescence lifetime imaging of oxygen, $\mathrm{pH}$ and carbon dioxide using optical sensors, Appl. Spectrosc. 54 (2000) 548-559.

[9] R.N. Glud, N.B. Ramsing, J.K. Gundersen, I. Klimant, Planar optrodes: a new tool for fine scale measurements of two-dimensional $\mathrm{O}_{2}$ distribution in benthic communities, Marine Ecol. Prog. Series 140 (1996) 217-226.

[10] R.N. Glud, M. Kühl, O. Kohls, N.B. Ramsing, Heterogeneity of oxygen production and consumption in a photosynthetic microbial mat as studied by planar optodes, J. Phycol. 35 (1999) 270-279.

[11] W.L. Rumsey, R. Iturriaga, D.F. Wilson, S. Lahiri, D. Spergel, Phosphorescence and Fluorescence Imaging: New Tools for the
Study of Carotid Body Function, Chemoreceptors and Chemoreceptor Reflexes, Plenum Press, New York, 1990, pp. 73-79.

[12] R.D. Shonat, D.F. Wilson, C.E. Riva, M. Pawlowski, Oxygen distribution in the retinal and choroidal vessels of the cat as measured by a new phosphorescence imaging method, Appl. Opt. 31 (1992) 3711-3718.

[13] S. Vinogradov, L.-W. Lo, W.T. Jenkins, S.M. Evans, C. Koch, D.F. Wilson, Non-invasive imaging of the distribution in oxygen in tissue in vivo using near-infrared phosphors, Biophys. J. 70 (1996) 16091617.

[14] S. Nomura, M. Nakao, T. Nakanishi, S. Takamastu, K. Tomita, Realtime imaging of microscopic $\mathrm{pH}$ distribution with a two-dimensional pH imaging apparatus, Anal. Chem. 69 (1997) 977-981.

[15] J.R. Lakowicz, I. Gryczynski, Frequency-Domain Fluorescence Spectroscopy, Topics in Fluorescence Spectroscopy: Technics 1, Plenum Press, New York, 1991, pp. 293-335.

[16] J.R. Lakowicz, H. Szmacinski, K. Nowaczyk, K.W. Berndt, M. Johnson, Fluorescence lifetime imaging, Anal. Biochem. 202 (1992) 316-330.

[17] P. Hartmann, W. Ziegler, Lifetime imaging of luminescent oxygen sensors based on all-solid-state technology, Anal. Chem. 68 (1996) $4512-4514$.

[18] M. Stücker, L. Schulze, G. Pott, P. Hartmann, D.W. Lübbers, A. Röchling, P. Altmeyer, FLIM of luminescencet oxygen sensors: clinical applications and results, Sens. Actuators B 51 (1998) $171-175$.

[19] R.N. Glud, J.K. Gundersen, N.P. Revsbech, B.B. Jørgensen, Effects on the benthic diffusive boundary layer imposed by microelectrodes, Limnology Oceanography 39 (1994) 462-467.

[20] C.G. Morgan, A.C. Mitchell, J.G. Murray, Fluorescence decay time imaging using an imaging photon detector with a radiofrequency photon correlation system, in: Proceedings of the Conference on Time-Resolved Laser Spectroscopy in Biochemistry II, 1990.

[21] R.M. Clegg, B. Feddersen, E. Gratton, T.M. Jovin, Time resolved imaging fluorescence microscopy, in: Proceedings of the Conference on Time-Resolved Laser Spectroscopy in Biochemistry II, 1992.

[22] X.F. Wang, T. Uchida, D.M. Coleman, S. Minami, A Twodimensional fluorescence lifetime imaging system using a gated image intensifier, Appl. Spectrosc. 45 (1991) 360-366.

[23] D.F. Wilson, S. Gomi, A. Pastuszko, J.H. Greenberg, Microvascular damage in the cortex of cat brain from middle cerebral artery occlusion and reperfusion, J. Appl. Phys. 74 (1993) 580-589.

[24] M. Gouterman, Oxygen quenching of luminescence of pressure sensitive paint for wind tunnel research, J. Chem. Education 74 (1997) 697-702.

[25] R.L. Plant, D.H. Burns, Quantitative, depth-resolved imaging of oxygen concentration by phosphorescence lifetime measurement, Appl. Spectrosc. 47 (1993) 1594-1599.

[26] C. Preininger, I. Klimant, O.S. Wolfbeis, Optical fiber sensor for biological oxygen demand (BOD), Anal. Chem. 66 (1994) 18411846.

[27] I. Klimant, P. Belser, O.S. Wolfbeis, in: Proceedings of the Conference on Novel Longwave Absorbing and Emitting Transition Metal Complexes for Use in Optical Oxygen Sensing, Europt(r)ode I, Graz, 1992.

[28] I. Klimant, V. Meyer, M. Kühl, Fiber-optic oxygen microsensors, a new tool in aquatic biology, Limnology Oceanography 40 (1995) $1159-1165$.

[29] I. Klimant, G. Holst, M. Kühl, Oxygen microoptodes and their application in aquatic environment, in: Proceedings of the SPIE Conference on Chemical Biochemical and Environmental Fibre Sensors VII, Vol. 2508, 1995, pp. 375-386.

[30] P. Hartmann, M.J.P. Leiner, Luminescence quenching behavior of an oxygen sensor based on a Ru(II) complex dissolved in polystyrene, Anal. Chem. 67 (1995) 88-93.

[31] P. Hartmann, W. Trettnak, Effects of polymer matrices on calibration 
functions of luminescent oxygen sensors based on porphyrine-ketone complexes, Anal. Chem. 68 (1996) 2615-2620.

[32] E.R. Carraway, J.N. Demas, B.A. DeGraff, J.R. Bacon, Photophysics and photochemistry of oxygen sensors based on luminescent transition metal complexes, Anal. Chem. 63 (1991) $337-342$.

[33] E.R. Carraway, J.N. Demas, B.A. DeGraff, Luminescence quenching mechanism for microheterogenous systems, Anal. Chem. 63 (1991) 332-336.

[34] G. Holst, R.N. Glud, M. Kühl, I. Klimant, A microoptode array for fine-scale measurement of oxygen distribution, Sens. Actuators B 38/ 39 (1997) 122-129.

[35] S.A. Vinogradov, D.F. Wilson, Metallotetrabenzoporphyrins: new phosphorescent probes for oxygen measurements, J. Chem. Soc., Perkin Trans. 2 (1995) 103-111.

[36] D.P. Papkovsky, J. Olah, I.V. Troyanovsky, N.A. Sadovsky, V.D. Rumyantseva, A.F. Mironov, A.I. Yaropolov, A.P. Savitsky, Phosphorescent polymer films for optical oxygen sensors, Biosens. Bioelectron. 7 (1991) 199-206.

[37] C. Huber, I. Klimant, C. Krause, O.S. Wolfbeis, Dual lifetime referencing (DLR) as applied to a chloride optical sensor, Anal. Chem., 1999, in press.

[38] O.S. Wolfbeis, I. Klimant, T. Werner, C. Huber, U. Kosch, C. Krause, G. Neurauter, A. Dürkop, Set of luminescence decay time based chemical sensors for clinical applications, Sens. Actuators B 51 (1998) 17-24.

[39] U. Kosch, I. Klimant, T. Werner, O.S. Wolfbeis, Strategies to design $\mathrm{pH}$ optodes with luminescence decay times in the microsecond time regime, Anal. Chem. 70 (1998) 3892-3897.

\section{Biographies}

Gerhard Holst, born in 1962, studied electronical engineering at the RWTH University in Aachen, Germany, where he received the diploma in 1990, with a final work about reflectance pulse oximetry with electrooptical and hybrid fiber optical sensors. From 1991 to 1994, he completed his $\mathrm{PhD}$ in the group of Prof. D.W. Lübbers at the Max-PlanckInstitute (MPI) for Molecular Physiology, Dortmund, Germany, about a new optical chemical sensing principle, the oxygen flux optode and its phase modulation based measuring system. From 1994 he joined the microsensor research group at the MPI for Marine Microbiology, Bremen, Germany, as a post-doc and scientist, where he works on the development of new fibre optical microsensors, microoptodes, new measuring systems and imaging systems for the application of planar optodes and their time resolved measuring schemes for laboratory and field applications.

Björn Grunwald, born in 1972, studied microsystems engineering/ microelectronics at the University of Bremen, Germany since 1992. He did his master thesis at the Max-Planck-Institute for Marine Microbiology (MPI); Bremen, Germany in 1999 about a fibre-optic microsensor for refractive index and salinity based on SPR. As a part of the course he worked on the feasibility of a $\mu$-microphone on the basis of a capacitive pressure sensor used for medical applications. From 1994-1995 he spent 1 year at the University of Bournemouth, England, studying microelectronics and computing. He worked at the National Microelectronics Research Centre, Cork, Ireland, resulting in a project for a micromachined resonatingplate immunosensor. In 2000 he continues his work on high spatial resolution fibre-optic refractive index sensors as a PhD student at the MPI. 